\title{
Original Non-alcoholic fatty liver disease among visitors to a

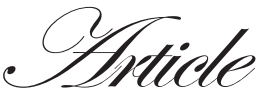 hepatitis awareness programme
}

\author{
Zaigham Abbas, ${ }^{1}$ Arzoo Saeed, ${ }^{1}$ Syed Mujahid Hassan, ${ }^{1}$ Nasir \\ Hassan Luck, ${ }^{1}$ Arsalan Khan, ${ }^{2}$ Mirza Naqi Zafar, ${ }^{3}$ Kehkashan Zehra \\ Hossain, Syed Muhammad Faiq
}

ABSTRACT

Departments of Hepatogastroenterology, ${ }^{1}$ Hepatobiliary Surgery, ${ }^{2}$ Pathology, ${ }^{3}$ Nutrition ${ }^{4}$ and Radiology, ${ }^{5}$ Sindh Institute of Urology and Transplantation, Karachi, Pakistan

\section{Correspondence:}

Dr Zaigham Abbas

Email:drzabbas@gmail.com
Background and aims: Non-alcoholic fatty liver disease (NAFLD) is rapidly becoming the most common liver disease worldwide. The present study aimed to evaluate the prevalence of NAFLD among the visitors screened on World Hepatitis Day 2010 and 2011.

Methods: Attendees of the hepatitis awareness programme $(n=928)$ were offered screening to rule out liver ailments. The participants were evaluated on history, body mass index (BMI) measurement, blood tests for hepatitis B surface antigen (HBsAg), anti-hepatitis C virus (anti-HCV), alanine aminotransferase (ALT) levels and abdominal ultrasound (US).

Results: On ultrasonography, fatty liver was present in 142 of 806 subjects who were found negative for viral serology and had no history of regular alcohol intake. This accounted for $15.3 \%$ of all the visitors. Comparing them with the rest of the serology-negative alcoholnegative subjects, there were more housewives $(\mathrm{p}=0.005)$ with fatty liver. They were older with mean age $43.3 \pm 12.1$ years compared to33.1 \pm 16.1 years $(\mathrm{p}=0.000)$ in patients without fatty liver and had a higher BMI $28.6 \pm 6.0 \mathrm{~kg} / \mathrm{m}^{2} \mathrm{vs} .22 .2 \pm 5.3 \mathrm{~kg} / \mathrm{m}^{2}(\mathrm{p}=0.000)$. Regression analysis suggested BMI as the only significant independent risk factor $(\mathrm{p}=0.000)$. However, 32 subjects with fatty liver had BMI $<25 \mathrm{~kg} / \mathrm{m}^{2}$ and 6 of them had diabetes mellitus. Thirty-one subjects $(21.8 \%)$ with fatty liver had raised ALT level $(\mathrm{p}=0.000)$ and possible non-alcoholic steatohepatitis (NASH) which accounts for 3.34\% (31/928) of the total visitors.

Conclusions: This analysis reveals that our general population is at risk of having NAFLD and NASH and these should be looked into even in non-obese persons.

KEYWORDS: non-alcoholic fatty live disease, non-alcoholic steatohepatitis, body mass index

\section{Introduction}

Non-alcoholic fatty liver disease (NAFLD) is increasingly being recognized as an important cause of chronic liver disease. It covers a wide spectrum of liver pathology_from simple steatosis through the necroinflammatory disorder of nonalcoholic steatohepatitis (NASH) leading to cirrhosis and hepatocellular carcinoma (HCC). ${ }^{1,2}$ Among patients with NASH, about $15 \%-20 \%$ will develop cirrhosis. ${ }^{3}$ The process is not related to excess alcohol intake but is associated with obesity, increased triglyceride levels and reduced high-density lipoprotein (HDL) cholesterol levels, diabetes mellitus (DM), insulin resistance (IR) and metabolic syndrome. ${ }^{4,5}$ NAFLD is rapidly becoming the most common liver disease worldwide 
with a prevalence of up to $30 \%$ in developed countries and nearly $10 \%$ in developing nations. ${ }^{6}$ In the USA where obesity is prevalent, it is estimated that approximately one-third of the general population has $\mathrm{NAFLD}^{7}$ and cirrhosis due to $\mathrm{NASH}$, which is one of the frequent reasons for liver transplantation. In Pakistan, the exact prevalence of NAFLD is not known. A study of individuals between 30 and 50 years of age showed that $13.5 \%$ visitors who came for annual check-up had elevated alanine aminotransferase (ALT) levels without any cause; this was presumed to be related to NAFLD. ${ }^{8}$

The World Hepatitis Day is observed globally to spread awareness about this silent killer. An elaborate programme was arranged at our institute on this day to increase public awareness and screen population for hepatitis. In addition to hepatitis B and C, we decided to screen our visitors for NAFLD. The aim of our study was to find the prevalence of NAFLD among the visitors to the hepatitis awareness programme.

\section{Methods}

Attendees of hepatitis awareness programmes held on World Hepatitis Day 2010 and 2011 were offered screening to rule out liver ailments. Informed consent was taken for participation in the screening programme. Trained volunteers who took history and collected data on a predesigned proforma interviewed the participants. The proforma included questions about demography, past history including liver disease, DM, hypertension, renal, cardiac or thyroid diseases, and risk factors for liver diseases. The exclusion criteria for the NAFLD study were known cases of any liver ailment; persons with a history of regular alcohol intake (a daily alcohol consumption $>20 \mathrm{~g}$ in women and $>30 \mathrm{~g}$ in $\mathrm{men}^{9}$ ); persons who were found infected with hepatitis $\mathrm{B}$ or $\mathrm{C}$ during the screening process; and patients known to have end-stage kidneys, congestive heart failure and hypothyroidism.

Vital parameters including blood pressure (BP), pulse and temperature of the participants were noted and height (in cm) and weight (in $\mathrm{kg}$ ) were checked by using the standard equipment. Body mass index (BMI) was calculated by dividing weight (in $\mathrm{kg}$ ) by height (in $\mathrm{m}^{2}$ ). Obesity and overweight were classified according to the World Health Organization (WHO) criteria. ${ }^{10}$ Abdominal ultrasound (US) was performed by certified radiologists using standard equipment (Samsung Medison, SonoAce). Abdominal scanning was performed in the supine position with breath-holding using a 5-7 $\mathrm{MHz}$ curvilinear probe. Liver echotexture was compared with that of spleen and kidney to make the diagnosis of fatty liver. Fatty liver was defined as the presence of at least two of the three abnormal findings on abdominal US - diffusely increased echogenicity ("bright") of the liver with liver echogenicity greater than that of kidney or spleen; vascular blurring; and deep attenuation of US signals. ${ }^{11-13}$ A hard copy image was obtained showing the liver and spleen parenchyma at the same US settings and anatomical plane.

Blood samples were taken for hepatitis B surface antigen (HBsAg), anti-hepatitis C virus (anti-HCV), and ALT levels. Hepatitis B and $\mathrm{C}$ screening tests were done by enzyme-linked immunosorbent assay (ELISA) method (Abbott ARCHITECT). Those persons who were found positive for hepatitis $\mathrm{B}$ or $\mathrm{C}$ or had elevated ALT levels and abnormal US were given appointments for follow-up at the clinic.

\section{Statistical analysis}

For descriptive statistics, frequency and percentage were used for categorical variables. For continuous variables, mean \pm standard deviation (SD) was used. Statistical analysis was performed using the Chi-square test with Yates correction for the comparison of discrete variables and Student t-test for the comparison of continuous variables. Levene's test was used before a comparison of means. When results of the Levene's test were significant, adjusted values were used that did not assume equality of variance. Risk factors for NAFLD found significant in univariate analysis were entered in the multivariate regression analysis to assess the variables independently associated with the presence of NAFLD. All analyses were done on computer package SPSS (Statistical Package for Social Science) version 19.0. In all statistical analysis, only p values $<0.05$ were considered significant.

\section{Results}

Among the 928 participants without any history of liver disease, $586(63.1 \%)$ were men and 342 (36.8\%) were women, with a mean age of $34.2 \pm 14.6$ years (range $1-93$ years). Seventeen $(1.76 \%)$ subjects were found out to be HBsAg-positive, 66 $(6.86 \%)$ were anti-HCV-positive and $2(0.2 \%)$ were coinfected with hepatitis B and C. Thirty-seven (7\%) serology-negative subjects had a history of regular alcohol intake leaving behind 806 subjects with non-regular use of alcohol. Among these, 


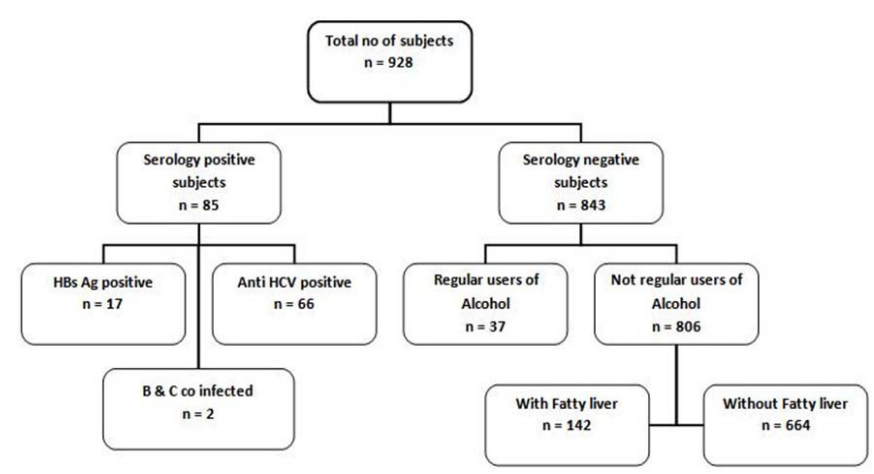

Figure 1: Flow diagram showing the characteristics of screened patients

Table 1: Comparison of characteristics in serology-negative, alcohol-negative subjects with or without fatty liver

\begin{tabular}{|c|c|c|c|}
\hline Risk factors & $\begin{array}{l}\text { Subjects with } \\
\text { fatty liver } \\
n=142\end{array}$ & $\begin{array}{l}\text { Subjects without } \\
\text { fatty liver } \\
n=664\end{array}$ & p-value \\
\hline $\begin{array}{l}\text { Age in years } \\
(\text { mean } \pm S D)\end{array}$ & $43.3 \pm 12.1$ & $33.1 \pm 16.1$ & 0.000 \\
\hline Gender (M:F) & $81: 61$ & $411: 253$ & 0.282 \\
\hline Married & 116 & 363 & 0.000 \\
\hline Housewives & 41 & 114 & 0.005 \\
\hline Hypertension & 12 & 33 & 0.323 \\
\hline Diabetes & 14 & 61 & 0.802 \\
\hline $\begin{array}{l}\text { Family history of } \\
\text { liver disease }\end{array}$ & 26 & 74 & 0.149 \\
\hline Weight (kg) & $75.2 \pm 6.0$ & $57.7 \pm 17.1$ & 0.000 \\
\hline Height $(\mathrm{cm})$ & $160.3 \pm 10.0$ & $157.0 \pm 16.7$ & 0.003 \\
\hline BMI $\left(\mathrm{kg} / \mathrm{m}^{2}\right)$ & $28.6 \pm 6.0$ & $22.2 \pm 5.3$ & 0.000 \\
\hline $\mathrm{BMI}>25 \mathrm{~kg} / \mathrm{m}^{2}$ & 110 & 196 & 0.000 \\
\hline $\begin{array}{l}\text { BMI }>23 \mathrm{~kg} / \mathrm{m}^{2} \\
\text { (Asia Pacific cut-off) }\end{array}$ & 125 & 288 & 0.000 \\
\hline Irregular liver surface & 4 & 9 & 0.211 \\
\hline $\begin{array}{l}\text { Increased portal vein } \\
\text { size }\end{array}$ & 15 & 54 & 0.812 \\
\hline Splenomegaly & 1 & 12 & 0.484 \\
\hline Gallstones & 4 & 9 & 0.437 \\
\hline $\begin{array}{l}\text { ALT level } \\
(\text { mean } \pm \text { SD) }\end{array}$ & $32.3 \pm 23.4$ & $27.7 \pm 20.1$ & 0.031 \\
\hline $\begin{array}{l}\text { Crossing ALT } \\
\text { upper limit }\end{array}$ & 31 & 87 & 0.026 \\
\hline $\begin{array}{l}\text { Crossing gender- } \\
\text { adjusted ALT limit }\end{array}$ & 72 & 288 & 0.111 \\
\hline
\end{tabular}

BMI=body mass index; ALT=alanine aminotransferase;

$\mathrm{SD}=$ standard deviation

fatty liver was found to be present in 142 subjects (15.3\% of the total visitors) on US (Figure 1).

Comparing patients with fatty liver with the rest of the serology-negative and alcohol-negative subjects, there were women with a large proportion of housewives comprising $29 \%$ of all the subjects with fatty liver $(\mathrm{p}=0.005)$. They were older with mean age $43.3 \pm 12.1$ years as compared to $33.1 \pm 16.1$ years

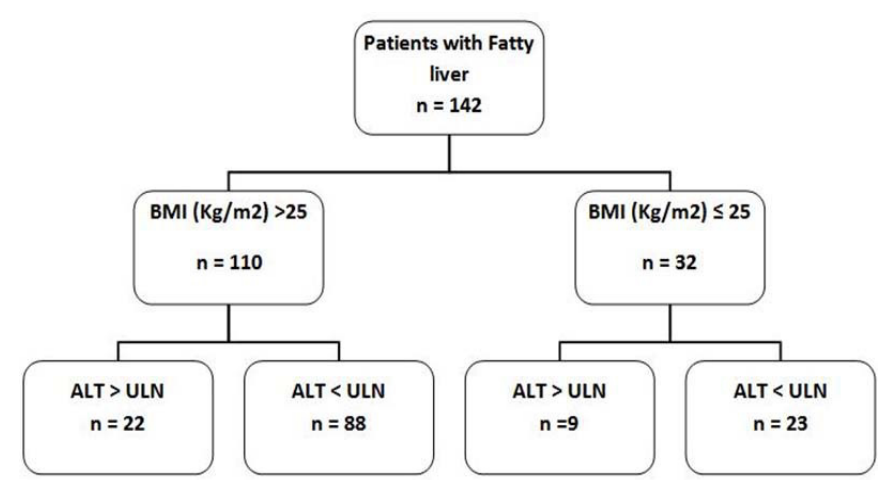

Figure 2: Body mass index (BMI) and alanine aminotransferase (ALT) levels in subjects with fatty liver

$(\mathrm{p}=0.000)$ in non fatty liver group and had a higher BMI of $28.6 \pm 6.0 \mathrm{~kg} / \mathrm{m}^{2}$ vs. $22.2 \pm 5.3 \mathrm{~kg} / \mathrm{m}^{2}(\mathrm{p}=0.000)$ (Table 1). Regression analysis suggested BMI as the only significant independent risk factor $(\mathrm{p}=0.000)$. However, 32 subjects with fatty liver had BMI $<25 \mathrm{~kg} / \mathrm{m}^{2}$ and 6 of them had diabetes mellitus (Figure 2). Thirty-one subjects (21.8\%) with fatty liver had elevated ALT level $(\mathrm{p}=0.000)$ and possible NASH which accounts for $3.34 \%$ (31/928) of the total visitors.

\section{Discussion}

NAFLD is a condition of excessive fat accumulation in the form of triglycerides (steatosis) in the liver in $>5 \%$ of hepatocytes histologically and this is not due to excess alcohol intake or other cause of steatosis. ${ }^{14}$ NAFLD is an increasingly common chronic liver disease worldwide and is associated with obesity, metabolic syndrome, dyslipidaemia, insulin resistance (IR) and type II DM. ${ }^{4}$ NAFLD is widely considered to be the liver expression of the metabolic syndrome which encompasses IR, central (truncal) obesity, hypertriglyceridaemia, low HDL cholesterol and hypertension. ${ }^{5}$

NAFLD is considered to be increasing in the Asia Pacific region including South Asia. Estimates of current prevalence in this region range from $5 \%$ to $30 \%$, depending upon the population studied. Central obesity, DM and metabolic syndrome are the major risk factors. ${ }^{15}$ Our study showed the occurrence of NAFLD in $15.3 \%$ of the apparently healthy visitors, which may be a reflection of its prevalence in the local population. Comparing South Asians with Caucasians for comparable BMI and age, healthy Asians have physiological markers for IR, dyslipidaemia, and increased cardiovascular risk. Alterations in body fat distribution, particularly increased abdominal and visceral fat, may contribute to these differences. ${ }^{16}$ 
A survey done among a Pakistani population of age range 25-64 years showed that $22.6 \%$ of women and $13.2 \%$ of men were overweight (BMI $\left.>25 \mathrm{~kg} / \mathrm{m}^{2}\right)$. Moving from low to middle to upper class the prevalence of NAFLD in rural areas was $9 \%$, $15 \%, 27 \%$, respectively and in urban areas it was $21 \%, 27 \%$, $42 \%$, respectively. This reflects the effects of industrialization and urbanization. ${ }^{17}$ Another study showed that the prevalence of overweight (BMIe" $23 \mathrm{~kg} / \mathrm{m}^{2}$ ) was $25.0 \%$ (95\% confidence interval [CI] 21.8\%--28.2\%) and that of obesity (BMI e"27 kg/ $\mathrm{m}^{2}$ ) was $10.3 \%$ (95\% CI $\left.7.0 \%-13.2 \%\right) .{ }^{18}$ The factors independently and significantly associated with overweight and obesity in local population included older age, being woman, urban residence, being literate, and having a high (versus low) economic status and a high (versus low) intake of meat. ${ }^{19}$ In our study, $38 \%$ of the subjects had BMI $>25$. The WHO has redefined the criteria of obesity for Asians. Hence obesity occurs when BMI is $>25 \mathrm{~kg} / \mathrm{m}^{2}$ and overweight when BMI is in the range $23-25 \mathrm{~kg} / \mathrm{m}^{2} .20,21$ According to these criteria, $38 \%$ of our serology-negative subjects were obese and the percentage of overweight with or without obesity increases to $51 \%$.

The Asia Pacific Working Party for NAFLD has incorporated the anthropometric measurements as regional criteria based on multiple Asian publications stressing on higher visceral fat and central obesity reflected by the waist-hip ratio (WHR). ${ }^{1}$ We could not measure the WHR due to time constraints of screening a large number of visitors in one day. Measuring waist circumference requires the persons to be adequately trained. The subject should be standing, and the circumference is determined at the midpoint between the lower margin of the rib cage and the iliac crest (in $\mathrm{cm}$ ). Failure to measure central obesity may account for NAFLD in our visitors with BMI $<25$ $\mathrm{kg} / \mathrm{m}^{2}$. We did not measure the parameters of metabolic syndrome such as triglyceride and HDL cholesterol level as it was not a part of the screening programme.

In our study, factors associated with NAFLD included age, BMI and being a housewife. Traditionally, in our culture housewives enjoy sedentary lifestyle and are responsible to look after cooking and kitchen so they are expected to develop fatty liver which is evident from our study. Customarily, NAFLD has been regarded as a benign disease; however, clinical as well as epidemiological studies had contradicted this belief and had shown that approximately $20 \%$ of patients with NAFLD had NASH..$^{1,2}$ In a study, the prevalence of NAFLD in patients with type II DM and metabolic syndrome in Pakistani population was found to be $72.4 \% .{ }^{22}$ In diabetic patients, the prevalence of NASH correlates with hypertriglyceridaemia, high ALT level and the prevalence of advanced fibrosis correlates with a high serum ã-glutamyl transferase level, older age and male gender. ${ }^{23}$ We could not find such associations probably due to fewer numbers of patients with DM in the cohort.

The majority of patients who seek medical consultation because of elevated levels of liver enzymes have metabolic syndrome and hence represent possible NASH. ${ }^{24}$ The NASH group constitutes the most common cause of cryptogenic cirrhosis. ${ }^{25}$ Hence about $5 \%-20 \%$ of patients with non-cirrhotic NASH would develop cirrhosis over a period of 10 years approximately. NASH-related cirrhosis is a risk factor for HCC. However, the occurrence of HCC is less common as compared to HCV cirrhosis. ${ }^{26-28}$ We used elevated ALT level as a criterion for NASH in individuals with NAFLD. However, one may have advanced fibrosis even in the presence of normal ALT levels.

Transaminase levels in NASH are typically elevated by less than five times of the upper limit of normal (ULN) and ALT or aspartate transferase (AST) value $>300 \mathrm{IU} / \mathrm{L}$ should raise suspicion of an alternative pathology. ${ }^{29}$ In our study, 31 subjects $(21.8 \%)$ with fatty liver had raised ALT level $(\mathrm{p}=0.000)$ and possible NASH which accounts for 3.34\% (31/928) of the total visitors. Recent studies suggest that ULN for serum ALT (generally between 40 and $55 \mathrm{IU} / \mathrm{L}$ ) required to be lowered to d"30 IU/L for men and d"19 IU/L for women. ${ }^{30}$ Though our patients with fatty liver had higher ALT levels and there were more subjects with elevated ALT level according to conventional cut-offs, we could not find any difference using the new genderadjusted cut-off values mentioned above. This may be related to lesser occurrence of fatty liver in the window between the old and the new cut-offs.

A study done by Niaz et al. ${ }^{8}$ showed that $13.5 \%$ of the individuals had elevated ALT levels without any cause and they were labelled as suffering from NAFLD. This study has many limitations and flaws in study design. The study included only men. Patients suffering from NAFLD were diagnosed only if elevated ALT levels were found without any cause. Individuals with normal ALT levels were not included in the study. The age of those included was restricted to fourth and fifth decade and high-risk individuals for NAFLD such as patients with DM and ischaemic heart disease were excluded from the study. US examination showed fatty change only in $86 / 129$ of the individuals labelled as patients with "NAFLD".

We used US abdomen to screen the subjects for NAFLD. US features of NAFLD include increased hepatic echogenicity, vascular blurring and deep attenuation of US signals. ${ }^{21}$ These 
three US features had good accuracy in detecting fatty liver and had good correlation with visceral obesity and metabolic syndrome. ${ }^{20,21}$ NAFLD diagnosis also requires other liver conditions to be excluded such as viral hepatitis (HBV, HCV) and alcoholic liver disease. ${ }^{1}$ We excluded these conditions by taking history and screening for HBV and HCV. However US is not the gold standard; and the precise diagnosis of NAFLD, distinction between hepatic steatosis and steatohepatitis and assessment of disease severity and degree of fibrosis requires a liver biopsy. ${ }^{25}$ Liver biopsy was not a part of our screening programme.

Subjects coming to a free medical camp for evaluation may not be entirely healthy. They may already be having an ailment and their health-seeking behaviour in a camp on awareness day may be a means to confirm or rule out their illness. This may introduce a bias in the evaluation and lead to overdiagnosis of a particular illness. So our study cannot reflect the true prevalence of NAFLD in the local population but does give a good estimate of the magnitude of the problem.

In Pakistan, obesity and lack of physical activity is on the rise. As a result, there is also a change in the prevalence of NAFLD in this region. This changing trend of liver disease is also seen in the rest of South Asia and the Asia Pacific region, reaching to an epidemic proportion of $30 \%$. Unhealthy lifestyle and poor health awareness is prevalent and may be aggravating this problem further. ${ }^{31}$ Our study highlights the importance of recognition of the fact that our general population is at risk of having NAFLD and NASH and these should be looked into even in non-obese individuals. We recommend that US abdomen should be included in the list of tests for persons undergoing health or pre-employment check-ups.

\section{References}

1. Chitturi S, Farrell GC, Hashimoto E, Saibara T, Lau GK, Sollano JD, Asia-Pacific Working Party on NAFLD. Nonalcoholic fatty liver disease in the Asia-Pacific region: definitions and overview of proposed guidelines. J Gastroenterology Hepatol. 2007;22:778-87.

2. Matteoni CA, Younossi ZM, Gramlich T, Boparai N, Liu YC, McCullough AJ. Nonalcoholic fatty liver disease: a spectrum of clinical and pathological severity. Gastroenterology. 1999;116:1413-19.

3. Sass DA, Chang P, Chopra KB. Nonalcoholic fatty liver disease: a clinical review. Dig Dis Sci. 2005;50:171-80.

4. Kotronen A, Yki-Jarvinen H, Mannisto S, Saarikoski L, KorpiHyovalti E, Oksa H, et al. Nonalcoholic and alcoholic fatty liver disease-two diseases of affluence associated with the metabolic syndrome and type 2 diabetes: the FIN-D2D survey. BMC Public Health. 2010;10:237.

5. Hamaguchi M, Kojima T, Takeda N, Nakagawa T, Taniguchi H, Fujii K, et al. The metabolic syndrome as a predictor of nonalcoholic fatty liver disease. Ann Intern Med. 2005; 143:722-8.

6. Smith BW, Adams LA. Nonalcoholic fatty liver disease. Crit Rev Clin Lab Sci. 2011;48:97-113.

7. Sanyal AJ. NASH: a global health problem. Hepatol Res. 2011;41:670-4.

8. Niaz A, Ali Z, Nayyar S, Fatima N. Prevalence of NAFLD in Healthy and Young Male Individuals. ISRN Gastroenterol. 2011;2011:363546.

9. Ratziu V, Bellentani S, Cortez-Pinto H, Day C, Marchesini G. A position statement on NAFLD/NASH based on the EASL 2009 special conference. J Hepatol. 2010;53:372-84.

10. World Health Organization. Physical status: the use and interpretation of anthropometry. Report of a WHO Expert Committee. World Health Organ Tech Rep Ser. 1995;854:1-452.

11. Kojima S, Watanabe N, Numata M, Ogawa T, Matsuzaki S. Increase in the prevalence of fatty liver in Japan over the past 12 years: analysis of clinical background. J Gastroenterol. 2003;38:954-61.

12. Yajima Y, Ohta K, Narui T, Abe R, Suzuki H, Ohtsuki M. Ultrasonographical diagnosis of fatty liver: significance of the liver-kidney contrast. Tohoku J Exp Med. 1983;139:43-50.

13. Farrell GC, Chitturi S, Lau GK, Sollano JD, Asia-Pacific Working Party on NAFLD. Guidelines for the assessment and management of nonalcoholic fatty liver disease in the Asia-Pacific region: executive summary. J Gastroenterol Hepatol. 2007;22:775-7.

14. Neuschwander-Tetri BA. Nonalcoholic steatohepatitis and the metabolic syndrome. Am J Med Sci. 2005;330:326-35.

15. Amarapurkar DN, Hashimoto E, Lesmana LA, Sollano JD, Chen PJ, Goh KL; Asia-Pacific Working Party on NAFLD. How common is nonalcoholic fatty liver disease in the Asia-Pacific region and are there local differences? J Gastroenterol Hepatol. 2007;22:788-93.

16. Raji A, Seely EW, Arky RA, Simonson DC. Body fat distribution and insulin resistance in healthy Asian Indians and Caucasians. $J$ Clin Endocrinol Metab. 2001;86:5366-71.

17. Pappas G, Akhtar T, Gergen PJ, Hadden WC, Khan AQ. Health status of the Pakistani population: a health profile and comparison with the United States. Am J Public Health. 2001;91:93-8.

18. WHO Expert Consultation. Appropriate body-mass index for Asian population and its implications for policy and intervention strategies. Lancet. 2004;363:157-63.

19. Jafar TH, Chaturvedi N, Pappas G. Prevalence of overweight and obesity and their association with hypertension and diabetes mellitus in an Indo-Asian population. CMAJ. 2006;175:1071-7.

20. World Health Organization. Definition, diagnosis and classification of diabetes mellitus and its complications-Part 1: diagnosis and classification of diabetes mellitus. Geneva: WHO, 1999;20-1.

21. Hamaguchi M, Kojima T, Itoh Y, Harano Y, Fujii K, Nakajima T, et al. The severity of ultrasonographic findings in nonalcoholic fatty liver disease reflects the metabolic syndrome and visceral fat accumulation. Am J Gastroenterol. 2007;102:2708-15. 
22. Butt AS, Hamid S, Jafri W, Salih M, Javed A. Prevalence and risk factors for NAFLD among native South Asian Pakistani patients with type II diabetes and metabolic syndrome. United European Gastroenterology Week. 2011: Abstract No.11-6979.

23. Leite NC, Villela-Nogueira CA, Pannain VL, Bottino AC, Rezende GF, Cardoso CR, et al. Histopathological stages of nonalcoholic fatty liver disease in type II diabetes: prevalences and correlated factors. Liver Int. 2011;31:700-6.

24. Clark JM, Brancati FL, Diehl AM. The prevalence and etiology of elevated aminotransferase levels in the United States. Am J Gastroenterol. 2003;98:960-7.

25. Preiss D, Sattar N. Nonalcoholic fatty liver disease: an overview of prevalence, diagnosis, pathogenesis and treatment considerations. Clin Sci (Lond). 2008;115:141-50.

26. Powell EE, Cooksley WG, Hanson R, Searle J, Halliday JW, Powell LW. The natural history of nonalcoholic steatohepatitis: a follow-up study of forty-two patients for up to 21 years. Hepatology. 1990;11:74-80.
27. Hui JM, Kench JG, Chitturi S, Sud A, Farrell GC, Byth K, et al. Long-term outcomes of cirrhosis in nonalcoholic steatohepatitis compared with hepatitis C. Hepatology. 2003;38:420-7.

28. Adams LA, Lymp JF, St Sauver J, Sanderson SO, Lindor KD, Feldstein A, et al. The natural history of nonalcoholic fatty liver disease: a population-based cohort study. Gastroenterology. 2005;129:113-21.

29. Ramesh S, Sanyal AJ. Evaluation and management of nonalcoholic steatohepatitis. J Hepatol. 2005;42:S2-S12.

30. Prati D, Taioli E, Zanella A, Della Torre E, Butelli S, Del Vecchio E, et al. Updated definitions of healthy ranges for serum alanine aminotransferase levels. Ann Intern Med. 2002;137:1-10.

31. Jafar TH, Hatcher J, Poulter N, Islam M, Hashmi S, Qadri Z, et al., Hypertension Research Group. Community-based interventions to promote blood pressure control in a developing country: a cluster randomized trial. Ann Intern Med. 2009;151:593-601. 